\title{
ANÁLISE DA ANGULAÇÃO DAS LÂMINAS MEDIAL E LATERAL DO PROCESSO PTERIGOIDE DO OSSO ESFENOIDE PELA TOMOGRAFIA COMPUTADORIZADA
}

\section{ANALYSIS OF THE ANGLE BETWEEN THE MEDIAL AND LATERAL BLADES OF THE PTERYGOID PROCESS OF THE SPHENOID BONE FOR COMPUTED TOMOGRAPHY}

\author{
Lílian Oliveira Salvador* \\ Ana Paula Marinho Moreira* \\ Matheus Saraiva* \\ Thásia Luis Dias Ferreira* \\ Maria José Albuquerque Pereira de Sousa e Tucunduva*
}

\begin{abstract}
RESUMO
Determinadas situações de alteração da normalidade envolvem a simetria óssea dos processos pterigoides como a hemi-hiperplasia que é uma anomalia de desenvolvimento caracterizada pelo crescimento assimétrico de uma ou mais partes do corpo. A relação entre o volume de músculos da mastigação e estruturas ósseas pode ser estudada em doentes com e sem assimetria facial. A importância do conhecimento da anatomia radiográfica para a interpretação de afecções ósseas foi embasada no conhecimento anatômico do padrão de normalidade, principalmente no tocante a estruturas que servem de inserção aos músculos da mastigação. Esta pesquisa tem por objetivo determinar da angulação das lâminas medial e lateral do processo pterigoide do osso esfenoide, por meio da tomografia computadorizada helicoidal. Neste trabalho, em uma amostra que contou com 52 exames de tomografias computadorizadas, foi mensurado o ângulo entre as lâminas medial e lateral do processo pterigoide do osso esfenoide. Foram obtidos ângulos com média de $41,33^{\circ}$ do lado D e $41,66^{\circ}$ do lado E.
\end{abstract}

DESCRITORES: Anatomia, Tomografia computadorizada, Processo pterigoide, Osso esfenoide.

\section{ABSTRACT}

The aim in this research is to determine the angle formed by the medial and lateral blades of the pterygoid process of the sphenoid bone through helical CT. Certain situations involve alteration of normal bone symmetry of the pterygoid processes as hemihyperplasia which is a developmental anomaly characterized by asymmetric growth of one or more body parts. The relationship between the volume of masticatoty muscles and bony structures may be studied in patients with and without facial asymmetry. The knowledge of radiographic anatomy for the interpretation of bone infections is based on knowledge of the normal anatomical structures especially regarding those that serve as suport to the masticatoty muscles. In this study, in 52 helical CT exames, the angle between the blades of the medial and lateral pterygoid process of the sphenoid bone were measured. The mean angles found were $41.33^{\circ}$ to the right side and $41.66^{\circ}$ to the left side.

DESCRIPTORS: Anatomy, Computed tomography, Pterygoid process, Sphenoid bone. 
SALVADOR LO

MOREIRA APM

SARAIVA M

FERREIRA TLD TUCUNDUVA MJAPS

ANÁLISE DA ANGULAÇÃO DAS LÂMINAS MEDIAL

E LATERAL

DO PROCESSO

PTERIGOIDE DO OSSO ESFENOIDE

PELA TOMOGRAFIA COMPUTADORIZADA

\section{6}

REV, ODONTOL,

UNIV. CID, SÃO

PAULO

$2013 ; 25(1): 25-$

30 , JAN-ABR

\section{N T R O D U ÇÃ O}

O músculo pterigoide medial origina-se na face medial da lâmina lateral do processo pterigoide e insere-se na face medial do ângulo e ramo da mandíbula. Tem como função a elevação da mandíbula $^{1,2}$.

O músculo pterigoide lateral, com fibras dispostas horizontalmente é o único músculo mastigador relacionado à articulação temporomandibular. Tem sua cabeça inferior inserida na face lateral da lâmina lateral do processo pterigoide e sua inserção distal na face anterior do disco articular e na fóvea do processo condilar da mandíbula. Tem como função a abertura da boca e protrusão da mandíbula ${ }^{1,2}$.

O conhecimento da anatomia imagenológica para a interpretação das estruturas ósseas e suas possíveis alterações é respaldado no conhecimento anatômico ósseo. Faz-se importante conhecer o que é tido por normal, e suas variações, para que seja possível reconhecer a presença de afecções. A radiografia é constituída de uma projeção bidimensional, originada pela superposição de estruturas tridimensionais, que podem se apresentar alteradas ou não em sua forma e dimensão. $\mathrm{O}$ diagnóstico diferencial entre imagens radiográficas de anatomia óssea normal e de processos patológicos deve ser feito baseado em conhecimentos prévios da anatomia radiográfica ${ }^{3}$.

\section{REVISÃO DA LITERATURA}

O músculo pterigoide medial é um espesso músculo quadrilátero que está fixado profundamente na face medial da lâmina lateral do processo pterigoide e na face sulcada do processo piramidal do osso palatino e, mais superficialmente, nas faces laterais do processo piramidal do osso palatino e na tuberosidade da maxila. Tem sua inserção na face medial do ângulo e ramo da mandíbula. Sua função é a elevação (oclusão) da mandíbula ${ }^{1,2}$.

O músculo pterigoide lateral é um músculo mais curto e espesso, em relação ao músculo pterigoide medial, sendo o único que se dispõe horizontalmente e também o único que se relaciona diretamente com a articulação temporomandibular. Com uma cabeça superior proveniente da superfície infratemporal e da crista da asa maior do osso esfenoide e uma cabeça inferior proveniente da face lateral da lâmina lateral do processo pterigoide, sua inserção ocorre na face anterior do disco articular (cabeça superior) e na fóvea do processo condilar da mandíbula (cabeça inferior). Sua função é abaixar, protruir e realizar movimentos de lateralidade na mandíbula1, ${ }^{1,2}$.

Em alguns casos, os músculos da mastigação podem estar relacionados a alterações no processo de mastigação ${ }^{4}$. O exame clínico de alguns desses músculos podendo constituir um desafio ao profissional. Em um estudo com 20 cadáveres, foi impossível palpar o músculo pterigoide lateral, em $100 \%$ dos espécimes, devido às estruturas anatômicas anexas e ao reduzido espaço dessa área, ficando, assim, inacessível à palpação intraoral. Portanto, segundo desses autores, esse procedimento deveria ser desconsiderado como método clínico de avaliação e dever-se-ia buscar alternativas para a análise funcional do músculo em questão.

Determinadas situações de alteração da normalidade envolvem a simetria óssea dos processos pterigoides como a hemi-hiperplasia, que é uma anomalia de desenvolvimento rara, caracterizada pelo crescimento acentuado e assimétrico de uma ou mais partes do corpo, sendo que essa condição poderia estar associada a uma síndrome ou não. Foram descritos dois tipos de hemi-hiperplasia: em um lado do corpo, denominada hemi-hiperplasia complexa, quando mais de um órgão estiver comprometido, ou um único órgão, no caso da hemi-hiperplasia simples. Quando o aumento for apenas de um lado da face, designa-se hiperplasia hemifacial. Essa condição foi descrita com maior frequência em mulheres (proporção de duas mulheres para cada homem), acometendo, em geral, o lado direito do cor$\mathrm{po}^{5}$.

Sugiyama et al. ${ }^{6}$ (2001) descreveram um caso de lipomatose associada a hiperplasia hemifacial, no qual consideraram a contribuição da ressonância magnética valorosa para o acompanhamento do desenvolvimento dessa afecção. Urban e 
Bruening $^{7}$ (2009) relataram um caso de um rapaz de 14 anos de idade, portador de hiperplasia hemifacial. Os autores salientaram que a imagem por ressonância magnética era particularmente adequada para detecção dessa condição. Em outro estudo, em 2010, Bou-Haidar et al. ${ }^{8}$ reforçaram a presença da lipomatose na hiperplasia hemifacial. Esses autores relataram uma predominância da lipomatose sobre estruturas de outros tecidos, observados tanto na ressonância magnética quanto na tomografia computadorizada. Em todos os estudos supracitados os tecidos apresentavam alterações representadas pelo ganho de volume das estruturas envolvidas.

Lee et al. ${ }^{9}$ (2001) afirmaram que a hiperplasia hemifacial era uma condição rara na qual ocorria um aumento unilateral do volume dos tecidos da face, tendo apresentado três casos nos quais apenas os músculos da expressão facial, estavam envolvidos. Kwon et al. ${ }^{10}$ (2007) e Pereira-Perdomo et al. ${ }^{11}$ (2010) também descreveram casos nos quais apenas os músculos foram acometidos, caracterizando a mio-hiperplasia hemifacial. Nesses casos, não houve relato de alteração nas estruturas ósseas que davam origem aos músculos da mastigação.

Uma possibilidade de alteração morfológica do processo pterigoide, que não se relaciona a alterações congênitas ou cromossômicas, é a fratura dessa estrutura que, embora raramente relatada, foi descrita por Eriksson e Håkansson ${ }^{12}$ (1979), em um caso no qual tal ocorrência acarretou problemas de má-oclusão, parestesia do nervo maxilar do lado afetado, o que sugeriu deslocamento do fragmento.

O estudo do padrão da normalidade das estruturas ósseas nas quais se inserem os músculos da mastigação, mais precisamente os músculos pterigoides medial e lateral, e a determinação das médias, no padrão de normalidade, dos ângulos formados entre as lâminas medial e lateral do processo pterigoide do osso esfenoide, permitiu analisar a possibilidade da presença de afecções relacionadas a essas estruturas e sugerir alterações do padrão mastigatório, uma vez constatada assimetria das mesmas, permitindo relacionar à situação de desequilíbrio muscular. Nes- te estudo, buscamos estabelecer a média das angulações formadas pelo encontro das lâminas medial e lateral do processo pterigoide do osso esfenoide, por meio da tomografia computadorizada helicoidal em situação de normalidade. Avaliou-se, ainda, a existência de desvios da média em função do lado, da idade e do gênero.

\section{METODOLOG I A}

Foram avaliados 52 cortes axiais de exames de tomografia computadorizada, provenientes de banco de imagens, constituindo uma mostra de conveniência, na qual foram mensurados os ângulos do lado direito e esquerdo, respectivamente, formados pelas lâminas medial e lateral do processo pterigoide do osso esfenoide. A análise de cada uma das imagens foi realizada em monitor de tela plana, nos laboratórios de informática da instituição, no visualizador de imagens, por três observadores distintos, constituindo um estudo cego. Esta pesquisa foi aprovada pelo Comitê de Ética em Pesquisa (CEP) da Universidade Cidade de São Paulo com o protocolo de pesquisa $n^{\circ} 13573452$ - CAAE 0026.0.186.000-11, sendo aprovada em 20 de abril de 2011.

Foi realizada uma análise estatística do tipo descritiva para avaliação das médias dos ângulos obtidos por cada observador e o teste de variância (ANOVA) e de regressão referente aos dados obtidos pela análise de três observadores, e os dados interobservadores foram cruzados utilizando-se o software Minitab 16, para lado, idade e gênero.

\section{RESULTADOS}

Foram encontrados ângulos com a média de $41,33^{\circ}$ do lado direito e $41,66^{\circ}$ do lado esquerdo. Foram encontrados ângulos para o observador 1 de $41,24^{\circ}$ à direita e $42,42^{\circ}$ à esquerda. O observador 2 encontrou $42,47^{\circ}$ à direita e $43,66^{\circ}$ à esquerda e o observador $339,84^{\circ}$ à direita e $41,74^{\circ}$ à esquerda. No tocante ao gênero, foi encontrada a média de $44,7^{\circ}$ para mulheres e 55,3 para homens.

\section{I SCUSSÃO}

A avaliação das estruturas musculoesqueléticas associadas aos músculos da
SALVADOR LO

MOREIRA APM

SARAIVA M

FERREIRA TLD

TUCUNDUVA MJAPS

ANÁLISE DA

ANGULAÇÃO DAS

LÂMINAS MEDIAL

E LATERAL

DO PROCESSO

PTERIGOIDE DO

OSSO ESFENOIDE

PELA TOMOGRAFIA

COMPUTADORIZADA
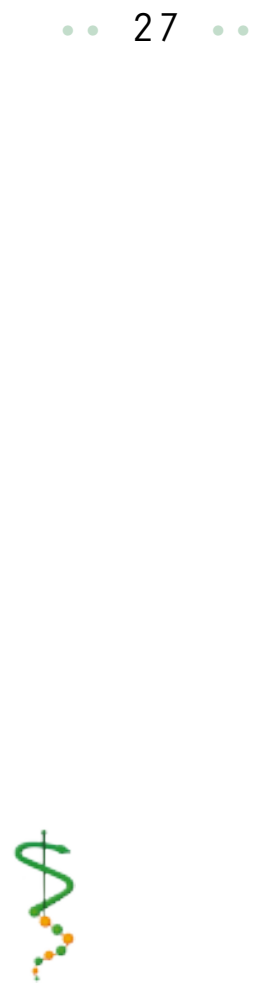

REV. ODONTOL.

UNIV, CID, SÃO

PAULO

2013; 25(1): 25-

30 , JAN-ABR 
SALVADOR LO

MOREIRA APM

SARAIVA M

FERREIRA TLD

TUCUNDUVA MJAPS

ANÁLISE DA ANGULAÇÃO DAS LÂMINAS MEDIAL

E LATERAL

DO PROCESSO

PTERIGOIDE DO

OSSO ESFENOIDE

PELA TOMOGRAFIA

COMPUTADORIZADA

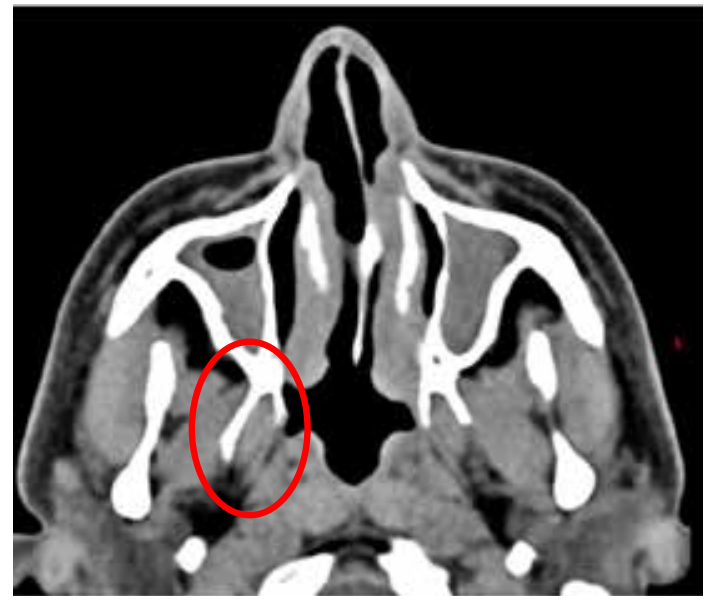

Fig. 1- Paciente gênero masculino, 32 anos com angulação medindo: Direita/40 e Esquerda/45

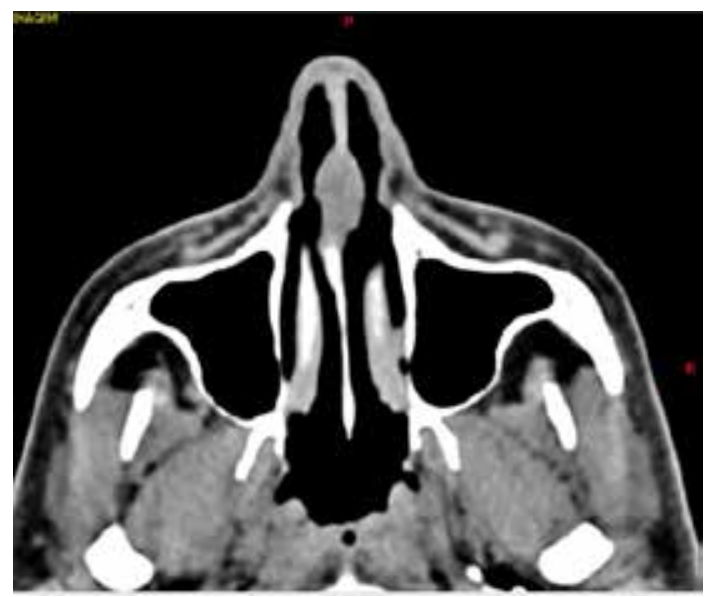

Fig. 2- Paciente gênero masculino, 61 anos com angulação medindo: Direita/40 e Esquerda/35

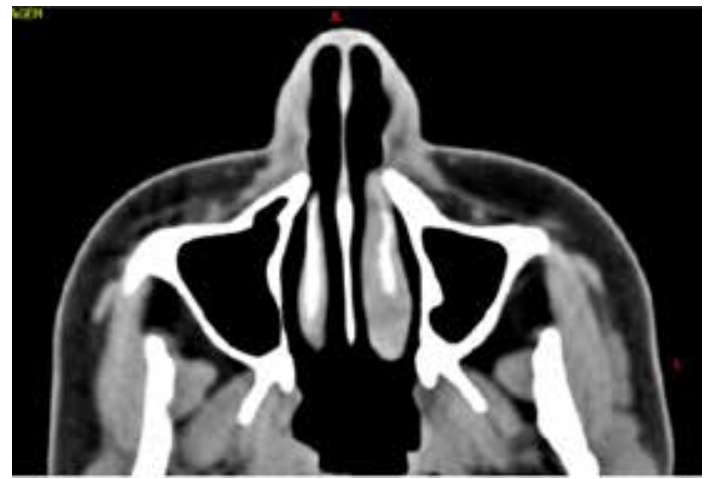

Fig. 3- Paciente gênero feminino, 20 anos, com angulação medindo: Direita/40 $\$$

REV, ODONTOL UNIV, CID, SÃO PAULO 2013; 25(1): 25 $30, J A N-A B R$

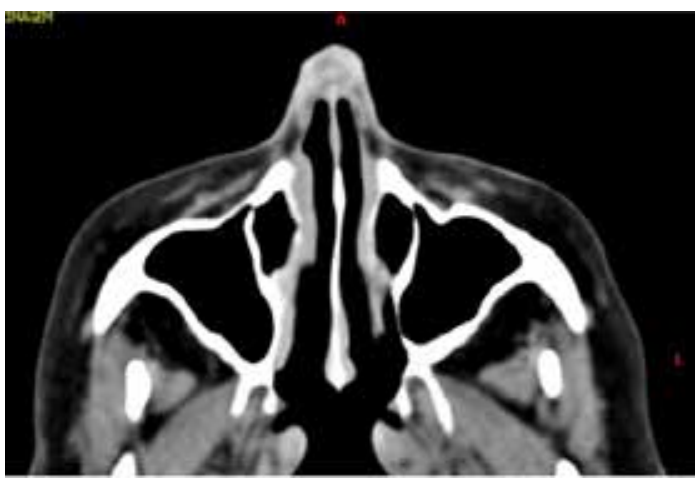

Fig. 4 - Paciente gênero feminino, 73 anos, com angulação medindo: Direita/ 56 e Esquerda/38

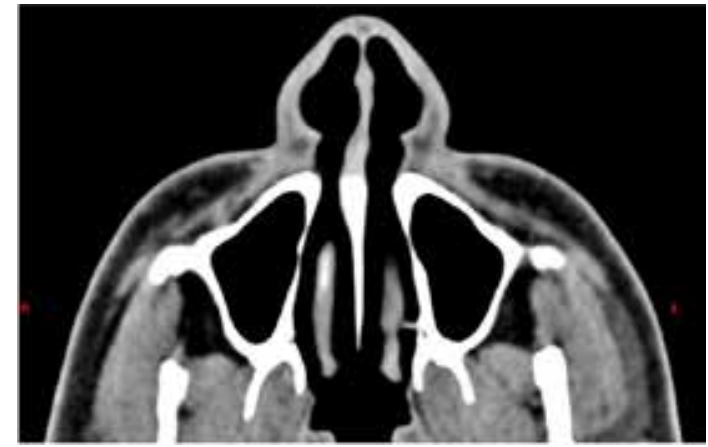

Fig. 5 - Paciente gênero masculino, 33 anos com angulação medindo: Direita/44 e Esquerda/44

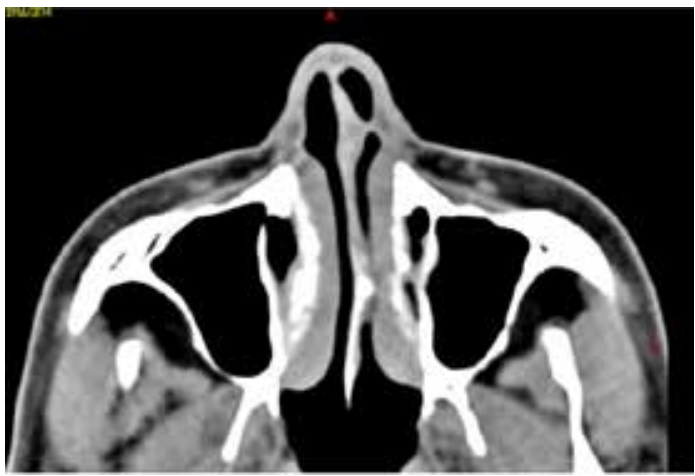

Fig. 6 - Paciente gênero masculino, 41anos com angulação medindo: Direita/45 e Esquerda/42

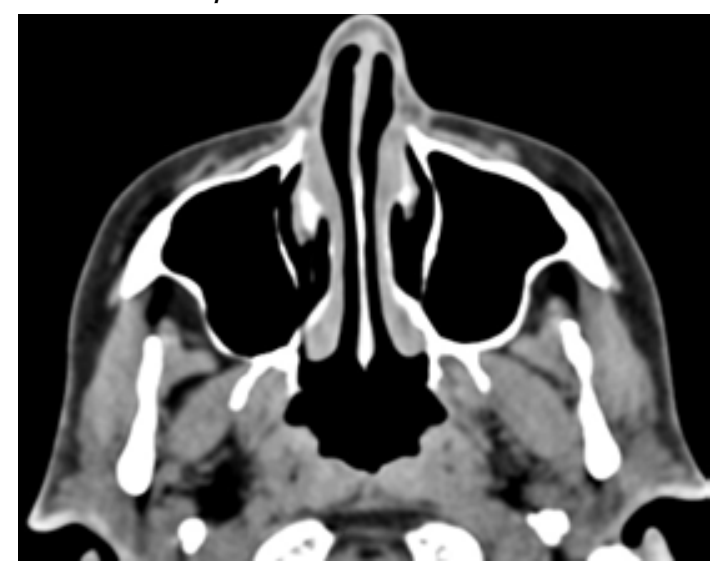

Fig. 7 - Paciente gênero feminino, 71 anos, com angulação medindo: Direita/ 50 e Esquerda/48 


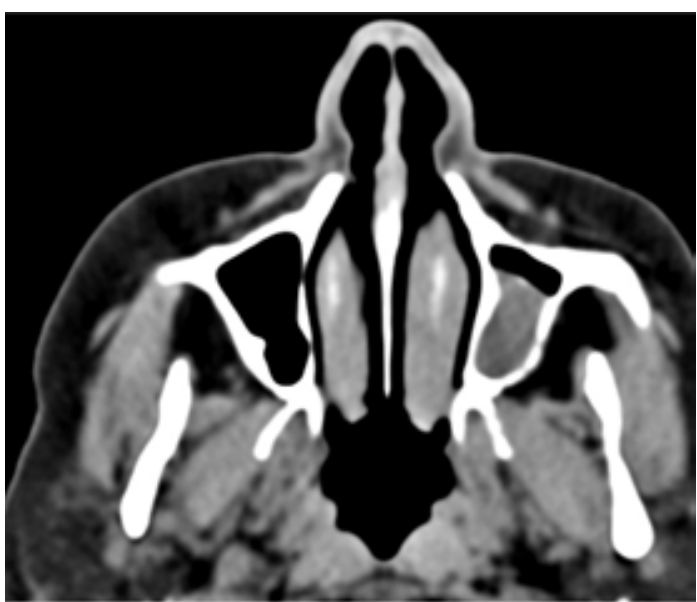

Fig. 8 - Paciente gênero feminino, 64 anos, com angulação medindo: Direita/ 40 e Esquerda/36

mastigação é dificultada pela sobreposição de estruturas adjacentes e pela dificuldade de acesso; entretanto, é fundamental que se possa estimar o grau de normalidade tanto de partes ósseas quanto de músculos relacionados ao processo de mastigação e, portanto, de oclusão para que se possa intervir, com segurança, sobre essas estruturas.

O diagnóstico por imagem, somado às características clínicas, pode ser de auxílio no estabelecimento de situações de normalidade, mas, para tanto, critérios como valores do padrão de normalidade devem ser estabelecidos para que sirvam de ponto de partida para avaliação das estruturas craniofaciais.

Antoniazzi et al. ${ }^{3}$ (2008) ressaltaram que o diagnóstico diferencial entre imagens radiográficas de anatomia óssea normal e de processos patológicos deve ser feito baseado em conhecimentos prévios da anatomia radiográfica. Vários auto- res, como Sugiyama et al. ${ }^{6}$ (2001); Urban e Bruening (2009); Bou-Haidar et al. ${ }^{8}$ (2010) apontam alterações das estruturas relacionadas com os músculos da mastigação, quer sejam dos músculos propriamente ditos, quer envolvam as partes ósseas que lhes servem de inserção, como no caso da hiperplasia hemifacial.

A complexidade das estruturas circunvizinhas e a limitação de espaço relacionado aos músculos pterigoides impedem que o exame clínico seja realizado de maneira apropriada, devendo o profissional lançar mão de recursos imagenológicos, como apontado por D'lppolito et al. ${ }^{4}$ (2010).

Este estudo buscou apresentar dados concernentes à condição de normalidade associada à origem proximal dos músculos pterigoides, que poderão ser utilizados como parâmetros iniciais na investigação de condições de assimetria facial, que possam ou não estar relacionadas às condições dentais e, portanto, de oclusão.

\section{CONCLUSÕES}

Após a avaliação dos dados obtidos, foi possível concluir que:

A média dos ângulos entre as lâminas medial e lateral do processo pterigoide do osso esfenoide em normalidade é de $41,33^{\circ}$ do lado direito e $41,66^{\circ}$ do lado esquerdo;

Não houve diferença dos ângulos com o aumento da idade;

Foram encontradas evidências de variação das médias, de acordo com o gênero, nos resultados de dois observadores, sendo a média de $44,7^{\circ}$ para mulheres e $55,3^{\circ}$ para homens.
SALVADOR LO MOREIRA APM SARAIVA M FERREIRA TLD

TUCUNDUVA MJAPS

ANÁLISE DA ANGULAÇÃO DAS LÂMINAS MEDIAL

E LATERAL

DO PROCESSO PTERIGOIDE DO OSSO ESFENOIDE

PELA TOMOGRAFIA COMPUTADORIZADA

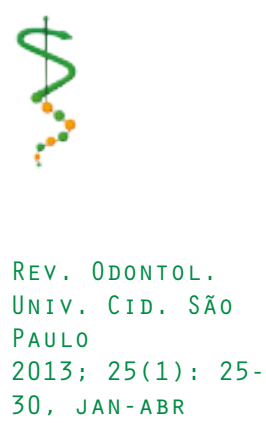


SALVADOR LO

MOREIRA APM

SARAIVA M :

FERREIRA TLD

TUCUNDUVA MJAPS

ANÁLISE DA

ANGULAÇÃO DAS

LÂMINAS MEDIAL

E LATERAL

DO PROCESSO

PTERIGOIDE DO

OSSO ESFENOIDE

PELA TOMOGRAFIA

COMPUTADORIZADA

\section{REFERÊNCIAS}

1. Williams PL. Gray anatomia. 37. ed. Rio de Janeiro: Guanabara koogan; 1995.

2. Rizzolo R, Madeira M. Anatomia Facial com fundamentos de anatomia sistêmica geral. 2. ed. São Paulo: Sarvier; 2006.

3. Antoniazzi MCC, Carvalho PL, Koide $\mathrm{CH}$. Importância do conhecimento da anatomia radiográfica para a interpretação de patologias ósseas RGO (Porto Alegre) 2008 abr.-jun.;56(2):195-9.

4. D'Ippolito SM, Borri Wolosker AM, D'Ippolito G, Herbert de Souza B, Fenyo-Pereira M. Evaluation of the lateral pterygoid muscle using magnetic resonance imaging. Dentomaxillofac Radiol 2010 Dec;39(8):494-500.

5. Neville B, Damm D, Allen C, Bouquot J. Patologia oral e maxilofacial. 2. ed. Rido de Janeiro: Guanabara Koogan; 2004.

6. Sugiyama M, Tanaka E, Ogawa I, Ishibashi R, Naito K, Ishikawa T. Magnetic resonance imaging in hemifacial hyperplasia. Dentomaxillofac Radiol 2001 Jul;30(4):235-8.

7. Urban PP, Bruening R. Congenital isolated hemifacial hyperplasia. J Neurol 2009 Sep;256(9):1566-9.

8. Bou-Haidar P, Taub P, Som P. Hemifacial lipomatosis, a possible subtype of partial hemifacial hyperplasia: CT and MR imaging findings. AJNR Am J Neuroradiol 2010 May;31(5):891-3.

9. Lee S, Sze R, Murakami C, Gruss J, Cunningham M. Hemifacial myohyperplasia: description of a new syndrome. Am J Med Genet 2001 Nov 1;103(4):326-33.

10. Kwon TG, Lee KH, Park HS, Ryoo HM, Kim HJ, Lee SH. Relationship between the masticatory muscles and mandibular skeleton in mandibular prognathism with and without asymmetry. J Oral Maxillofac Surg 2007 Aug;65(8):1538-43.

11. Pereira-Perdomo DF, Velez-Forero J, Prada-Madrid R. Hemifacial myohyperplasia sequence. Am J Med Genet $A 2010$ Jul;152A(7):1770-3.

12. Eriksson L, Hakansson $\mathrm{H}$. Unilateral fracture of the pterygoid process. Report of a case. Oral Surg Oral Med Oral Pathol 1979 Feb;47(2):127-30.

Recebido em: 07/10/2012

Aceito em: 04/12/2012
REV, ODONTOL,

UNIV. CID. SÃO

PAULO

2013; 25(1): $25-$

30 , JAN-ABR: 